\title{
PRKCDBP (CAVIN3) and CRY2 associate with major
} depressive disorder

Kovanen, Leena

2017-01

Kovanen , L , Donner , K , Kaunisto , M \& Partonen , T 2017 , ' PRKCDBP (CAVIN3) and CRY2 associate with major depressive disorder ' , Journal of Affective Disorders , vol. 207 , pp. 136-140 . https://doi.org/10.1016/j.jad.2016.09.034

http://hdl.handle.net/10138/230037

https://doi.org/10.1016/j.jad.2016.09.034

publishedVersion

Downloaded from Helda, University of Helsinki institutional repository.

This is an electronic reprint of the original article.

This reprint may differ from the original in pagination and typographic detail.

Please cite the original version. 
Research paper

\title{
PRKCDBP (CAVIN3) and CRY2 associate with major depressive disorder
}

\author{
Leena Kovanen $^{\mathrm{a}, *}$, Kati Donner ${ }^{\mathrm{b}}$, Mari Kaunisto ${ }^{\mathrm{b}}$, Timo Partonen ${ }^{\mathrm{a}}$ \\ a Department of Health, National Institute for Health and Welfare (THL), Helsinki, Finland \\ ${ }^{\mathrm{b}}$ Institute for Molecular Medicine Finland (FIMM), University of Helsinki, Helsinki, Finland
}

\section{A R T I C L E I N F O}

\section{Keywords:}

Diurnal

Genetic association

Health examination

Population

\begin{abstract}
A B S T R A C T
Background: Dysfunctions in the intrinsic clocks are suggested in patients with depressive disorders. The cryptochrome circadian clocks 1 and 2 (CRY1 and CRY2) proteins modulate circadian rhythms in a cell and influence emotional reactions and mood in an individual. The protein kinase $\mathrm{C}$ delta binding protein (PRKCDBP, or CAVIN3), similar to the serum deprivation response protein (SDPR, or CAVIN2), reduces metabolic stability of the PER2-CRY2 transcription factor complex that plays a role in the circadian rhythm synchronization. Our aim was to study SDPR, PRKCDBP, CRY1 and CRY2 genetic variants in depressive disorders.

Methods: The sample included 5910 Finnish individuals assessed with the Munich-Composite International Diagnostic Interview (M-CIDI) in year 2000. In year 2011, 3424 individuals were assessed again. After genotype quality control, there were 383 subjects with major depressive disorder, 166 with dysthymia, and 479 with depressive disorders (major depressive disorder, dysthymia or both), and 4154 healthy controls. A total of 48 single-nucleotide polymorphisms from $S D P R, P R K C D B P, C R Y 1$ and $C R Y 2$ genes were analyzed using logistic regression models controlling for age and gender.

Results: The earlier reported association of $C R Y 2$ variants with dysthymia was confirmed and extended to major depressive disorder $(q<0.05)$. In addition, novel associations of PRKCDBP rs1488864 with depressive disorders $(\mathrm{q}=0.02)$ and with major depressive disorder in specific $(\mathrm{q}=0.007)$ were found.

Limitations: The number of cases was moderate and coverage of PRKCDB was limited.

Conclusions: CRY2 and PRKCDBP variants may be risk factors of major depressive disorder and provide information for diagnosis.
\end{abstract}

\section{Introduction}

Patients with depressive disorders often have not only sleep disturbances, but also abnormalities in their sleep-wakefulness cycle or rapid-eye-movement sleep that suggest there are dysfunctions in the intrinsic clocks generating and maintaining the circadian rhythms. Concerning the circadian rhythms, a strong repressor during the evening is irreplaceable to the morning phase in the normal circadian clock (Ukai-Tadenuma et al., 2008). The cryptochrome circadian clocks 1 and 2 (CRY1 and CRY2) are strong repressors, and their endogenous properties confer information as a function of time (Edwards et al., 2016). A delay in transcription of $C R Y 1$ via clock-controlled DNA elements from the $C R Y 1$ promoter and a further delay via clockcontrolled DNA elements from the $C R Y 1$ intron are the steps that keep the amplitude of circadian clocks robust (Ukai-Tadenuma et al., 2011). Therefore, CRY1 and CRY2 are plausible targets of interest. Here, CRY2 has a key role, as it not only acts as a general repressor, but also opposes the actions of CRY1 and inhibits CRY1 from accessing to its
DNA targets too early, possibly facilitated by PER proteins (Anand et al., 2013). Repression by CRY1 is like a homeostatic process with an autonomous and open-ended action that extends from circadian night into circadian day. It is the ratio rather than the absolute amounts of CRY proteins that determines the circadian period, and, e.g., if the CRY1-to-CRY2 ratio is greater than it is usually is, then the nuclear import rate of the PER-CRY complex slows down and the circadian period lengthens (Li et al., 2016). Further, it is only CRY2 however that can inhibit the activated forms of ARNTL, the key clock protein, while the CRY1 and PER proteins have no effect (Dardente et al., 2007). Intriguingly, as the levels of PER2 in the correct phase is far more critical for the circadian synchronization than PER2 levels as such, the dimerization of PER2 with CRY1 or with CRY2 may play a role in the pathogenesis (Chen et al., 2009). It is of note that the interval $\varphi$ (alignment difference) tracks dawn and dusk, and that for the measurement of alignment the CRY1 and CRY2 proteins give a signal of the evening hours, whereas the PER1 and PER2 proteins give a signal of the morning hours in day-active animals, e.g. in sheep

\footnotetext{
* Correspondence to: National Institute for Health and Welfare, P.O. Box 30, FI-00271 Helsinki, Finland.

E-mail address: leena.kovanen@thl.fi (L. Kovanen).
} 
(Lincoln et al., 2003).

Currently, it is not known, whether the aforementioned dynamics between PER and CRY proteins holds for humans as well. Here, if it were to hold, the model built by Aziz Sancar and his coworkers (Ye et al., 2011, 2014) may provide a key to understanding of the pathogenesis in mood disorders. The model says that the CRY proteins are in a dynamic equilibrium with the ARNTL-CLOCK complex on Eboxes, that in the presence of PER proteins at a sufficiently high concentration in the nucleus all CRY proteins become trapped by the dimerization, and that these dimers cannot bind to the ARNTL-CLOCK complex on E-boxes (Ye et al., 2011, 2014). Thus, a key to the pathogenesis may lie in abnormal signals of the evening that are due to the actions of the CRY1 and CRY2 proteins encoded by CRY1 and $C R Y 2$, respectively, whose genetic variants might therefore associate with major depressive disorder. Moreover, the CRY1 and CRY2 proteins block the increases in cyclic adenosine monophosphate, which are driven by activation of $\mathrm{G}$ protein-coupled receptors, e.g. those for dopamine, and the subsequent cascades in the liver (Zhang et al., 2010) and possibly universally within the organism (O’Neill et al., 2008). It has been demonstrated that the increases in cyclic adenosine monophosphate and the downstream activation of cyclic-adenosine-monophosphate-responsive element binding protein in the striatum lead to a depressive-like behavioral phenotype in mice (Park et al., 2005). To sum up, the CRY1 and CRY2 proteins are hence strategically positioned to modulate not only circadian rhythms of a cell or a tissue but also to influence emotional reactions and mood of the individual.

Beyond, the protein kinase $\mathrm{C}$ delta binding protein (PRKCDBP, also known as CAVIN3) regulates the abundance of PER2 and possibly CRY2 (Schneider et al., 2012). PRKCDBP increases the strength of interaction within the PER2-CRY2 complex and reduces their metabolic stability, similar to the serum deprivation response (SDPR, also known as CAVIN2), and the CAVIN3 gain-of-function lengthens the circadian period.

Thus, genes $S D P R, P R K C D B P, C R Y 1$, and $C R Y 2$ are among the key targets for studying the etiology and genetic mechanisms of mood disorders. The aim of our present work was therefore to study SDPR and $P R K C D B P$ genes in major depressive disorder and dysthymia (chronic depressive disorder). In addition, with more cases identified in the year 2011, we wanted to confirm the positive association of CRY2 with dysthymia reported earlier.

\section{Methods}

\subsection{Subjects}

Our sample included 5910 individuals who had in year 2000 given blood samples, taken part to the Munich-Composite International Diagnostic Interview (M-CIDI) (Wittchen et al., 1998) and filled in a self-report on the seasonal variations in mood and behavior adapted from the Seasonal Pattern Assessment Questionnaire (Rosenthal et al., 1984). Of these 5910 individuals, 3424 took part in the M-CIDI interview again in year 2011. The sample is part of the national Health 2000 survey (Aromaa and Koskinen, 2004) of the Finnish population aged 30 years and older $(n=8028)$ and its follow-up survey in the year $2011(n=7964)$. The study was approved by the ethics committees of the National Public Health Institute and the Helsinki and Uusimaa Hospital District. The study was carried out in accordance with the principles of the Declaration of Helsinki and its amendments. All participants provided a written informed consent.

\subsection{Phenotypes}

Phenotypes of interest were major depressive disorder, dysthymia, and depressive disorders (herein, major depressive disorder, dysthymia, or both). Cases included the participants diagnosed to have a depressive disorder in year 2000 added with new cases from year 2011.
Healthy controls were free of depressive disorders, anxiety disorders (panic disorder w/o agoraphobia, generalized anxiety disorder, social phobia, and/or agoraphobia) and alcohol use disorders (abuse and/or dependence) in year 2000 and also in year 2011, if they took part in the follow-up. For depressive and anxiety disorders, the diagnoses were assessed for the past 12 months and for alcohol use disorders for the lifetime.

\subsection{Gene and SNP selection}

Selection of SNPs in SDPR, PRKCDBP, CRY1 and CRY2 was based on HapMap phase 3 data (http://www.hapmap.org/), and tagging was done using the Tagger program in the Haploview 4.1 software (Barrett et al., 2005). The linkage disequilibrium (LD) within the gene and within $10 \mathrm{~kb}$ of their $5^{\prime}$ and $3^{\prime}$ flanking regions, that is, $122 \mathrm{~kb}$ for CRY1 (chr12: 107 375-107 $497 \mathrm{~kb}$, GRCh37/hg19 assembly), $56 \mathrm{~kb}$ for CRY2 (chr11:45 859-45 $915 \mathrm{~kb}$ ), $22 \mathrm{~kb}$ for PRKCDBP (chr11: 6329-6351 kb) and $33 \mathrm{~kb}$ for SDPR (chr2:192 689-192 722), was used to select tag-SNPs capturing most of the genetic variation. The aim was to capture all the SNPs having a minor allele frequency (MAF) of $>5 \%$ in the European population (CEU and TSI) in the HapMap database. The pair-wise $\mathrm{r}^{2}$ was set to $\geq 0.9$ in order to select a tag-SNP among the SNPs that were in high LD. Ten out of $21 C R Y 1,10$ out of $34 C R Y 2$ and 3 out of 14 SDPR SNPs fulfilled the criterion and were all successfully included in the genotyping multiplexes. Of the 12 out of 19 PRKCDBP SNPs fulfilling the criterion, 8 were successfully included. In addition to the aforementioned tag-SNPs, 20 potentially functional $C R Y 1$ (12) and $C R Y 2$ (8) variants were selected using Pupasuite,21 Variowatch,22 database of SNPs affecting miR Regulation (dbSMR)23 and microRNA SNP24 databases, and were included in the study. Supplementary Table S1 presents all the 53 SNPs that were successfully genotyped in this study.

\subsection{Genotyping}

Genomic DNA was isolated from whole blood according to standard procedures. The SNPs were genotyped at the Institute for Molecular Medicine Finland (FIMM), Technology Centre, University of Helsinki, using the MassARRAY iPLEX method (Sequenom, San Diego, CA, USA) (Jurinke et al., 2002), with excellent success (>95\%) and accuracy (100\%) rates (Lahermo et al., 2006). For quality control purposes, positive (CEPH) and negative water controls were included in each 384-plate. Genotyping was performed blind to phenotypic information.

314 of 5910 individuals were excluded due to a high missing genotype rate (i.e. $>0.1$ ). The total genotyping rate in the remaining individuals was 0.997 . Three SNPs turned out to be non-polymorphic (CRY2 rs35488012, rs117531403 and rs76545099), and two SNPs were removed because their minor allele frequency was $<0.01$ (CRY2 rs3747548, CRY1 rs7294758). Finally, there were 5596 individuals and 48 SNPs for the statistical analyses.

\subsection{Statistical analyses}

SNP and haplotype statistical analyses were performed using logistic regression and additive genetic model controlling for age and gender (unadjusted models in Supplementary Table S3 online) with PLINK software version 1.07 (Purcell et al., 2007). Only haplotypes with more than $5 \%$ frequency are reported. In order to account for multiple comparisons, false discovery rate (FDR) q-values (Storey, 2003) were computed to correct for the SNPs and phenotypes analyzed using stats package in R software version 3.0.0 (R Core Team, 2015).

\section{Results}

General characteristics of the participants are shown in Table 1, and 
Table 1

General characteristics of the participants.

\begin{tabular}{|c|c|c|c|}
\hline & Major depressive disorder & Dysthymia & Depressive disorder \\
\hline $\mathrm{n}$ cases 2000 & 253 & 121 & 328 (46 both diagnosis) \\
\hline n reassessed in 2011 (\%) & $164(64.8)$ & $73(60.3)$ & $206(62.8)$ \\
\hline male gender \% & 30.8 & 31.4 & 30.5 \\
\hline age 2000 (mean \pm s.d. [range]) & $47.2 \pm 11.9[30-81]$ & $53.3 \pm 13.8[30-88]$ & $48.8 \pm 12.7[30-88]$ \\
\hline n cases 2011 & 161 & 58 & 201 (18 both diagnosis) \\
\hline male gender \% & 28.6 & 37.9 & 31.8 \\
\hline age 2011 (mean \pm s.d. [range]) & $54.6 \pm 9.9[41-93]$ & $54.8 \pm 10.3[41-84]$ & $54.9 \pm 10.0[41-93]$ \\
\hline $\mathrm{n}$ all cases & 383 & 166 & 479 (70 both diagnosis) \\
\hline male gender \% & 29.8 & 33.7 & 30.7 \\
\hline age 2000 & $46.2 \pm 11.4[30-82]$ & $50.6 \pm 13.7[30-88]$ & $47.4 \pm 12.2[30-88]$ \\
\hline n controls all & 4154 (2456 reassessed in 2011) & 4154 (2456 reassessed in 2011) & $4154(2456$ reassessed in 2011$)$ \\
\hline male gender $\%$ & 43.2 & 43.2 & 43.2 \\
\hline age $2000($ mean \pm s.d. [range]) & $53.5 \pm 14.8[30-97]$ & $53.5 \pm 14.8[30-97]$ & $53.5 \pm 14.8[30-97]$ \\
\hline
\end{tabular}

Table 2

Significant (q-value < 0.05) SNP associations of the adjusted model.

\begin{tabular}{|c|c|c|c|c|c|c|c|c|c|}
\hline Phenotype & Gene & SNP & $\mathrm{A} 1$ & $\mathrm{~N}$ & OR & L95 & U95 & $\mathrm{P}$-value & q-value \\
\hline Dysthymia & $C R Y 2$ & rs7121611 & $A$ & 4318 & 1.59 & 1.28 & 1.99 & 0.00004 & 0.002 \\
\hline Dysthymia & $C R Y 2$ & rs10838524 & $G$ & 4317 & 1.56 & 1.25 & 1.95 & 0.00009 & 0.003 \\
\hline Dysthymia & $C R Y 2$ & rs7945565 & $G$ & 4308 & 1.59 & 1.28 & 1.99 & 0.00004 & 0.002 \\
\hline Dysthymia & $C R Y 2$ & rs1401419 & $G$ & 4306 & 1.59 & 1.27 & 1.98 & 0.00004 & 0.002 \\
\hline Dysthymia & $C R Y 2$ & rs3824872 & $T$ & 4319 & 0.61 & 0.46 & 0.82 & 0.001 & 0.02 \\
\hline Major depressive disorder & $P R K C D B P$ & rs1488864 & $A$ & 4527 & 1.58 & 1.24 & 2.01 & 0.0002 & 0.01 \\
\hline Major depressive disorder & $C R Y 2$ & rs7123390 & $A$ & 4535 & 0.77 & 0.65 & 0.92 & 0.004 & 0.05 \\
\hline Major depressive disorder & $C R Y 2$ & rs2292910 & $A$ & 4534 & 0.78 & 0.67 & 0.93 & 0.004 & 0.05 \\
\hline Depressive disorders & PRKCDBP & rs1488864 & $A$ & 4623 & 1.47 & 1.17 & 1.84 & 0.0009 & 0.02 \\
\hline Depressive disorders & CRY2 & rs7121611 & $A$ & 4631 & 1.25 & 1.09 & 1.43 & 0.002 & 0.02 \\
\hline Depressive disorders & $C R Y 2$ & rs7945565 & $G$ & 4619 & 1.25 & 1.09 & 1.43 & 0.001 & 0.02 \\
\hline Depressive disorders & CRY2 & rs1401419 & $G$ & 4619 & 1.24 & 1.08 & 1.42 & 0.002 & 0.03 \\
\hline Depressive disorders & CRY2 & rs2292910 & $A$ & 4630 & 0.79 & 0.68 & 0.92 & 0.002 & 0.03 \\
\hline
\end{tabular}

A1, Tested allele (minor allele).

$\mathrm{N}$, Number of individuals included in analysis.

OR, Odds ratio.

L95/U95, Upper/Lower bounds of 95\% confidence interval.

the genotype frequencies, minor allele frequencies and HardyWeinberg equilibrium (HWE) p-values in the whole sample are reported in Supplementary Table 1 online (please, see Supplementary Tables S2-S4 online for the genotype counts of major depressive disorder, dysthymia and depressive disorders, respectively). All the single-nucleotide polymorphisms (SNPs) were in $\operatorname{HWE}(\mathrm{P}>0.01)$. Table 2 displays the significant $(\mathrm{q}<0.05)$ SNP association results of the adjusted model. In brief, one PRKCDBP SNP (rs1488864) associated both with major depressive disorder $(\mathrm{OR}=1.6,95 \% \mathrm{CI}=1.2-2.0$, $\mathrm{p}=0.0002, \mathrm{q}=0.007)$ and with depressive disorders (OR=1.5, 95\% $\mathrm{CI}=1.2-1.8, \mathrm{p}=0.0009, \mathrm{q}=0.02)$. Seven CRY2 SNPs associated with dysthymia, major depressive disorder, or depressive disorders. All the SNP association results are shown in Supplementary Tables S5 and S6 online (adjusted and unadjusted models, respectively).

The haplotype blocks formed are shown in Table 3, the significant $(\mathrm{q}<0.05)$ adjusted haplotype associations in Table 4 , and all haplotype associations in Supplementary Table S7 online. PRKCDBP haplotype ATTA (rs1488864|rs2947030|rs16911940|rs10839553) associated with the increased odds for major depressive disorder $(\mathrm{OR}=1.6$, $\mathrm{p}=0.0003, \mathrm{q}=0.02)$ and depressive disorders (OR=1.5, $\mathrm{p}=0.001$, $\mathrm{q}=0.02$ ). $C R Y 2$ haplotype $A T G C G G G G C A C G$ (rs7121611|rs7121775| rs10838524|rs2292913|rs7945565|rs1401419|rs7123390| rs4755345|rs17787136|rs10838527|rs2292910|rs3824872) associated with dysthymia $(\mathrm{OR}=1.5, \mathrm{p}=0.0004, \mathrm{q}=0.02)$.

\section{Discussion}

$C R Y 2$ rs10838524 $G$ allele, rs7121611 $A$ allele, rs7945565 $G$ allele, rs1401419 $G$ allele and rs3824872 $G$ allele were associated with
Table 3

Haplotype blocks estimated.

\begin{tabular}{|c|c|c|c|}
\hline Gene & $\mathrm{KB}$ & NSNPs & SNPs \\
\hline$S D P R$ & 14.4 & 2 & rs7577607|rs10174723 \\
\hline$P R K C D B P$ & 8.5 & 4 & rs1488864|rs2947030|rs16911940|rs10839553 \\
\hline CRY2 & 41.5 & 12 & $\begin{array}{l}\text { rs7121611|rs7121775|rs10838524|rs2292913| } \\
\text { rs7945565|rs1401419|rs7123390|rs4755345| } \\
\text { rs17787136|rs10838527|rs2292910|rs3824872 }\end{array}$ \\
\hline$C R Y 1$ & 4.7 & 3 & rs4964513|rs714359|rs12821586 \\
\hline CRY1 & 92.2 & 15 & $\begin{array}{l}\text { rs11113153|rs3741892|rs10861688|rs10861695| } \\
\text { rs10861697|rs2078074|rs59790130|rs10437895| } \\
\text { rs10746077|rs11613557|rs2888896|rs11113179| } \\
\text { rs10746083|rs4964518|rs10778528 }\end{array}$ \\
\hline
\end{tabular}

$\mathrm{KB}$, the kilobase distance spanned by the block.

NSNPs, number of SNPs in the block.

dysthymia, which was supported by haplotype analysis. CRY2 rs7123390 $G$ allele and $\operatorname{rs} 2292910 C$ allele were associated with major depressive disorder. CRY2 rs7121611 A allele, rs7945565 $G$ allele, rs1401419 $G$ allele and rs2292910 $C$ allele were associated with depressive disorders. PRKCDBP rs1488864 $A$ allele was associated both with major depressive disorder and with depressive disorders (depressive disorders, dysthymia or both), which were supported by haplotype analysis.

We have earlier reported four of the SNPs (CRY2 SNPs rs10838524, rs7121611, rs7945565 and rs1401419) to associate with dysthymia (Kovanen et al., 2013). During the 11-year follow-up period, the number of individuals with dysthymia increased from 136 to 186. The fifth CRY2 tag-SNP rs3824872 now associated with dysthymia is 
Table 4

Significant (q-value $<0.05$ ) haplotype associations of the adjusted model.

\begin{tabular}{|c|c|c|c|c|c|c|c|c|c|}
\hline Gene & Phenotype & NSNPs & SNP1 & SNP2 & Haplotype & $\mathrm{F}$ & OR & P-value & q-value \\
\hline CRY2 & Dysthymia & 12 & rs7121611 & rs3824872 & $A T G C G G G G C A C G$ & 0.45 & 1.48 & 0.0004 & 0.02 \\
\hline$P R K C D B P$ & Major depressive disorder & 4 & rs1488864 & rs 10839553 & ATTA & 0.08 & 1.57 & 0.0003 & 0.02 \\
\hline$P R K C D B P$ & Depressive disorders & 4 & rs1488864 & rs10839553 & ATTA & 0.08 & 1.46 & 0.001 & 0.02 \\
\hline
\end{tabular}

NSNPs, number of SNPs in the haplotype.

SNP1, 5' SNP.

SNP2, 3' SNP.

$\mathrm{F}$, haplotype frequency.

OR, estimated odds ratio.

located downstream of $C R Y 2$ gene and upstream of MAPK8IP1 gene. Earlier, we have also reported the associations of rs10838524 allele $G$ and rs7123390 allele $G$ in a Finnish sample, and those of rs10838524 allele $A, \operatorname{rs} 10838527$ allele $G$ and rs3824872 allele $A$ in a Swedish sample with winter depression (Lavebratt et al., 2010). In addition, $C R Y 2$ rs10838524 $A$ allele has been associated with bipolar disorder with rapid cycling, and $C R Y 2$ haplotype GGAC (of SNPs rs10838524, rs7123390, rs10838527 and rs3824872) was under-represented among the rapid-cycling cases (Sjoholm et al., 2010). Furthermore, CRY2 rs10838524 $A$ allele has been associated with greater chronicity of depressive symptoms (Fiedorowicz et al., 2012), and weak associations of $C R Y 2$ rs10838524 $G$ allele, and of haplotype $G G$ of rs 10838524 and rs7123390 with depressed female patients with early morning awakening have been reported (Utge et al., 2010). However, there are also reports of no association of CRY2 rs10838524 with major depressive disorder (Hua et al., 2014) and of no association of rs7121611 and rs2292910 with major depressive disorder or bipolar disorder (Soria et al., 2010).

Concerning CRY2, there is currently no information whether the SNPs we analyzed have any functional relevance to mood or behavior. Of the 12 SNPs forming the dysthymia-associated haplotype, however, five (two in specific, i.e., rs10838524 and rs3824872) were significantly associated with dysthymia, two (one in specific, i.e., rs7123390) with major depressive disorder, and four (i.e., rs7121611, rs7945565, rs1401419 and rs2292910) with depressive disorders (herein, dysthymia, major depressive disorder, or both). To have an idea for a mechanistic hypothesis of the impact of these genetic variants on the clinical phenotypes, a search from a bioinformatics database (HaploReg, v4.1, www.broadinstitute.org) yielded some information about these SNPs as follows. CRY2 rs10838524 as an intronic SNP influences the binding of the glucocorticoid receptor (NR3C1) which is implicated in depressive disorders (Goodwin et al., 1992) and known to interact reciprocally with CRY2 (Lamia et al., 2011; Torres-Farfan et al., 2009), thus having an impact on physiology and possibly on mood regulation as well. CRY2 rs10838524 also influences the binding of the CCCTC-binding factor (CTCF) and may thereby have an effect on transcriptional regulation of $C R Y 2$.

With regard to $P R K C D B P$, this is the first time that polymorphisms within this gene are linked to depressive disorders. The protein has earlier been shown to affect circadian clock functions (Schneider et al., 2012): PER2 complex interacts with PRKCDBP, and PER-to-CRY protein abundance and interactions are affected by PRKCDBP. In addition, the circadian period length is influenced by PRKCDBP such that the loss-of function shortened the period (Schneider et al., 2012), similar to knockout animal models of each key clock gene except that of Cry2 (Ko and Takahashi, 2006). Cry2 knockout mice have a prolonged circadian period which as such resembles the gain-of-function for PRKCDBP that is the lengthened circadian period. A search from the aforementioned bioinformatics database yielded that PRKCDBP rs1488864 influences the binding of 10 proteins, among them NFKB1 which is known to interact with cryptochromes (Lee and Sancar, 2011; Narasimamurthy et al., 2012) and implicated in depressive disorders (Miklowitz et al., 2016) but with conflicting data (Mellon et al., 2016), and BCL11A which is relevant to a characterized at-risk phenotype for anxiety and depressive disorders (Alisch et al., 2014).

Our study has several strengths. We based our study on a large random sample representative of the Finnish adult population and its 11-year follow-up data. The diagnoses of mental health were assessed with a structured and validated diagnostic interview method which yielded reliable diagnoses of depressive disorders based on the DSM-IV classification. We had well-covered genetic information for variants in both cryptochrome genes and SDPR gene. We applied strict multiple statistical testing correction by the false-discovery rate method. Despite the increased number of cases from the follow-up, Finland is still a relatively small country by population and therefore the number of cases remains moderate. Further, some SNPs in PRKCDB were not successfully included in the genotyping multiplexes.

To conclude, our study supports the role of $C R Y 2$ in depressive disorders and reports a novel association of $P R K C D B P$ with major depressive disorder.

\section{Funding}

This research did not receive any specific grant from funding agencies in the public, commercial, or not-for-profit sectors.

\section{Author contributions}

T.P. and L.K. designed the study. M.K. and K.D. designed and conducted the genotyping. L.K. performed statistical analysis. All authors interpreted the results and participated to manuscript preparation and approved its final version.

\section{Competing financial interests statement}

None to report.

\section{Appendix A. Supplementary material}

Supplementary data associated with this article can be found in the online version at doi:10.1016/j.jad.2016.09.034.

\section{References}

Alisch, R.S., Chopra, P., Fox, A.S., Chen, K., White, A.T., Roseboom, P.H., Keles, S., Kalin, N.H., 2014. Differentially methylated plasticity genes in the amygdala of young primates are linked to anxious temperament, an at risk phenotype for anxiety and depressive disorders. J. Neurosci. 34, 15548-15556.

Anand, S.N., Maywood, E.S., Chesham, J.E., Joynson, G., Banks, G.T., Hastings, M.H., Nolan, P.M., 2013. Distinct and separable roles for endogenous CRY1 and CRY2 within the circadian molecular clockwork of the suprachiasmatic nucleus, as revealed by the Fbxl3Afh mutation. J. Neurosci. 33, 7145-7153.

AromaaA.KoskinenS., 2004. Health and functional capacity in Finland. Baseline Results of the Health 2000 Health Examination Survey. Kansanterveyslaitoksen julkaisuja B: $12 / 2004$.

Barrett, J.C., Fry, B., Maller, J., Daly, M.J., 2005. Haploview: analysis and visualization of LD and haplotype maps. Bioinformatics 21, 263-265.

Chen, R., Schirmer, A., Lee, Y., Lee, H., Kumar, V., Yoo, S.H., Takahashi, J.S., Lee, C., 2009. Rhythmic PER abundance defines a critical nodal point for negative feedback 
within the circadian clock mechanism. Mol. Cell. 36, 417-430.

Dardente, H., Fortier, E.E., Martineau, V., Cermakian, N., 2007. Cryptochromes impair phosphorylation of transcriptional activators in the clock: a general mechanism for circadian repression. Biochem. J. 402, 525-536.

Edwards, M.D., Brancaccio, M., Chesham, J.E., Maywood, E.S., Hastings, M.H., 2016. Rhythmic expression of cryptochrome induces the circadian clock of arrhythmic suprachiasmatic nuclei through arginine vasopressin signaling. Proc. Natl. Acad. Sci. U. S. A 113, 2732-2737.

Fiedorowicz, J.G., Coryell, W.H., Akhter, A., Ellingrod, V.L., 2012. Chryptochrome 2 variants, chronicity, and seasonality of mood disorders. Psychiatr. Genet. 22, 305-306.

Goodwin, G.M., Muir, W.J., Seckl, J.R., Bennie, J., Carroll, S., Dick, H., Fink, G., 1992. The effects of cortisol infusion upon hormone secretion from the anterior pituitary and subjective mood in depressive illness and in controls. J. Affect. Disord. 26, 73-83.

Hua, P., Liu, W., Chen, D., Zhao, Y., Chen, L., Zhang, N., Wang, C., Guo, S., Wang, L., Xiao, H., Kuo, S.H., 2014. Cry1 and Tef gene polymorphisms are associated with major depressive disorder in the Chinese population. J. Affect. Disord. 157, $100-103$.

Jurinke, C., van den Boom, D., Cantor, C.R., Koster, H., 2002. Automated genotyping using the DNA MassArray technology. Methods Mol. Biol. 187, 179-192.

Ko, C.H., Takahashi, J.S., 2006. Molecular components of the mammalian circadian clock. Hum. Mol. Genet. 15 (2), R271-R277.

Kovanen, L., Kaunisto, M., Donner, K., Saarikoski, S.T., Partonen, T., 2013. CRY2 genetic variants associate with dysthymia. PLoS One 8, e71450.

Lahermo, P., Liljedahl, U., Alnaes, G., Axelsson, T., Brookes, A.J., Ellonen, P., Groop, P.H., Hallden, C., Holmberg, D., Holmberg, K., Keinanen, M., Kepp, K., Kere, J., Kiviluoma, P., Kristensen, V., Lindgren, C., Odeberg, J., Osterman, P., Parkkonen, M., Saarela, J., Sterner, M., Stromqvist, L., Talas, U., Wessman, M., Palotie, A., Syvanen, A.C., 2006. A quality assessment survey of SNP genotyping laboratories. Hum. Mutat. 27, 711-714.

Lamia, K.A., Papp, S.J., Yu, R.T., Barish, G.D., Uhlenhaut, N.H., Jonker, J.W., Downes, M., Evans, R.M., 2011. Cryptochromes mediate rhythmic repression of the glucocorticoid receptor. Nature 480, 552-556.

Lavebratt, C., Sjoholm, L.K., Soronen, P., Paunio, T., Vawter, M.P., Bunney, W.E. Adolfsson, R., Forsell, Y., Wu, J.C., Kelsoe, J.R., Partonen, T., Schalling, M., 2010. CRY2 is associated with depression. PLoS One 5, e9407.

Lee, J.H., Sancar, A., 2011. Regulation of apoptosis by the circadian clock through NFkappaB signaling. Proc. Natl. Acad. Sci. U. S. A 108, 12036-12041.

Li, Y., Xiong, W., Zhang, E.E., 2016. The ratio of intracellular CRY proteins determines the clock period length. Biochem. Biophys. Res. Commun. 472, 531-538.

Lincoln, G.A., Andersson, H., Hazlerigg, D., 2003. Clock genes and the long-term regulation of prolactin secretion: evidence for a photoperiod/circannual timer in the pars tuberalis. J. Neuroendocrinol. 15, 390-397.

Mellon, S.H., Wolkowitz, O.M., Schonemann, M.D., Epel, E.S., Rosser, R., Burke, H.B., Mahan, L., Reus, V.I., Stamatiou, D., Liew, C.C., Cole, S.W., 2016. Alterations in leukocyte transcriptional control pathway activity associated with major depressive disorder and antidepressant treatment. Transl. Psychiatry 6, e821.

Miklowitz, D.J., Portnoff, L.C., Armstrong, C.C., Keenan-Miller, D., Breen, E.C., Muscatell, K.A., Eisenberger, N.I., Irwin, M.R., 2016. Inflammatory cytokines and nuclear factor-kappa B activation in adolescents with bipolar and major depressive disorders. Psychiatry Res. 241, 315-322.

Narasimamurthy, R., Hatori, M., Nayak, S.K., Liu, F., Panda, S., Verma, I.M., 2012. Circadian clock protein cryptochrome regulates the expression of proinflammatory cytokines. Proc. Natl. Acad. Sci. USA 109, 12662-12667.
O'Neill, J.S., Maywood, E.S., Chesham, J.E., Takahashi, J.S., Hastings, M.H., 2008. cAMP-dependent signaling as a core component of the mammalian circadian pacemaker. Science 320, 949-953.

Park, S.K., Nguyen, M.D., Fischer, A., Luke, M.P., Affar el, B., Dieffenbach, P.B., Tseng, H.C., Shi, Y., Tsai, L.H., 2005. Par-4 links dopamine signaling and depression. Cell $122,275-287$.

Purcell, S., Neale, B., Todd-Brown, K., Thomas, L., Ferreira, M.A., Bender, D., Maller, J., Sklar, P., de Bakker, P.I., Daly, M.J., Sham, P.C., 2007. PLINK: a tool set for wholegenome association and population-based linkage analyses. Am. J. Hum. Genet. 81, $559-575$.

R Core Team, 2015. R: A language and environment for statistical computing. R Foundation for Statistical Computing, Vienna, Austria. URL 〈https://www.R-project. $\operatorname{org} /\rangle$.

Rosenthal, N., Bradt, G., Wehr, T., 1984. Seasonal Pattern Assessment Questionnaire (SPAQ). National Institute of Mental Health, Bethesda, Maryland.

Schneider, K., Kocher, T., Andersin, T., Kurzchalia, T., Schibler, U., Gatfield, D., 2012. CAVIN-3 regulates circadian period length and PER: cry protein abundance and interactions. EMBO Rep. 13, 1138-1144.

Sjoholm, L.K., Backlund, L., Cheteh, E.H., Ek, I.R., Frisen, L., Schalling, M., Osby, U., Lavebratt, C., Nikamo, P., 2010. CRY2 is associated with rapid cycling in bipolar disorder patients. PLoS One 5, e12632.

Soria, V., Martinez-Amoros, E., Escaramis, G., Valero, J., Perez-Egea, R., Garcia, C., Gutierrez-Zotes, A., Puigdemont, D., Bayes, M., Crespo, J.M., Martorell, L., Vilella, E., Labad, A., Vallejo, J., Perez, V., Menchon, J.M., Estivill, X., Gratacos, M., Urretavizcaya, M., 2010. Differential association of circadian genes with mood disorders: cry1 and NPAS2 are associated with unipolar major depression and CLOCK and VIP with bipolar disorder. Neuropsychopharmacology 35, 1279-1289.

Storey, J.D., 2003. The positive false discovery rate: a Bayesian Interpretation and the qValue. Ann. Stat. 31, 2013-2035.

Torres-Farfan, C., Abarzua-Catalan, L., Valenzuela, F.J., Mendez, N., Richter, H.G., Valenzuela, G.J., Seron-Ferre, M., 2009. Cryptochrome 2 expression level is critical for adrenocorticotropin stimulation of cortisol production in the capuchin monkey adrenal. Endocrinology 150, 2717-2722.

Ukai-Tadenuma, M., Kasukawa, T., Ueda, H.R., 2008. Proof-by-synthesis of the transcriptional logic of mammalian circadian clocks. Nat. Cell Biol. 10, 1154-1163.

Ukai-Tadenuma, M., Yamada, R.G., Xu, H., Ripperger, J.A., Liu, A.C., Ueda, H.R., 2011. Delay in feedback repression by cryptochrome 1 is required for circadian clock function. Cell 144, 268-281.

Utge, S.J., Soronen, P., Loukola, A., Kronholm, E., Ollila, H.M., Pirkola, S., PorkkaHeiskanen, T., Partonen, T., Paunio, T., 2010. Systematic analysis of circadian genes in a population-based sample reveals association of TIMELESS with depression and sleep disturbance. PLoS One 5, e9259.

Wittchen, H.U., Lachner, G., Wunderlich, U., Pfister, H., 1998. Test-retest reliability of the computerized DSM-IV version of the Munich-Composite International Diagnostic Interview (m-CIDI). Soc. Psychiatry Psychiatr. Epidemiol. 33, 568-578.

Ye, R., Selby, C.P., Ozturk, N., Annayev, Y., Sancar, A., 2011. Biochemical analysis of the canonical model for the mammalian circadian clock. J. Biol. Chem. 286, 25891-25902.

Ye, R., Selby, C.P., Chiou, Y.Y., Ozkan-Dagliyan, I., Gaddameedhi, S., Sancar, A., 2014. Dual modes of CLOCK: bmal1 inhibition mediated by Cryptochrome and Period proteins in the mammalian circadian clock. Genes Dev. 28, 1989-1998.

Zhang, E.E., Liu, Y., Dentin, R., Pongsawakul, P.Y., Liu, A.C., Hirota, T., Nusinow, D.A., Sun, X., Landais, S., Kodama, Y., Brenner, D.A., Montminy, M., Kay, S.A., 2010. Cryptochrome mediates circadian regulation of cAMP signaling and hepatic gluconeogenesis. Nat. Med. 16, 1152-1156. 